\title{
Fixed Point and Subfixed Point for Fuzzy Mappings in Generalized Metric Fuzzy Spaces
}

\author{
Mila Stojaković, ${ }^{1}$ Ljiljana Gajić, ${ }^{2}$ and Biljana Carićc \\ ${ }^{1}$ Department of Mathematics, Faculty of Technical Sciences, University of Novi Sad, Novi Sad, Serbia \\ ${ }^{2}$ Department of Mathematics, Faculty of Science, University of Novi Sad, Novi Sad, Serbia \\ Correspondence should be addressed to Mila Stojaković; stojakovic@sbb.rs
}

Received 20 August 2013; Accepted 13 November 2013

Academic Editor: Shih-sen Chang

Copyright (c) 2013 Mila Stojaković et al. This is an open access article distributed under the Creative Commons Attribution License, which permits unrestricted use, distribution, and reproduction in any medium, provided the original work is properly cited.

A generalized metric in space of set of fuzzy sets is introduced. We prove some common fixed point for contractive iterate at the point and orbitally contractive at the point fuzzy mappings and subfixed point results for family of mappings satisfying generalized contractive conditions in generalized metric fuzzy spaces.

\section{Introduction}

Uncertainty regarding some experiments may essentially have two origins. It may arise from randomness due to the natural variability of observation or it may be caused by imprecisions due to partial information, for example, expert opinions or sparse data sets. An incomplete data set delivers an imprecise assessment of the information which should be expressed by a $[0,1]$-fuzzy set instead of a number. In other words, the system is complemented with extra dimension of uncertainty provided by fuzzy set theory. Fuzzy logic is the principal component of an array of methodologies for dealing with problems in which uncertainty and imprecision play important roles.

Fixed point theory in uncertain systems can be treated in different ways and one of them is by using the fuzzy logic. Depending on which segment of the problem is plagued with some sort of uncertainty the appropriate structure of the basic space in which the problem is considered could be used. If the distance between elements is imprecise, then the fuzziness is included in metric, as it was done in the definition of fuzzy metric spaces by Kaleva and Seikkala [1]. This model has a lot of similarities with probabilistic metric spaces (Menger spaces). Fixed point techniques, methods, and results are closely related in these two structures. Some of fixed point results in fuzzy metric space could be found in [2-7].
If affiliations of elements are imprecise, then the system could be treated as a fuzzy system. Appropriate structure is introduced depending on the related problem. The treatment of the problem involving fixed point theory has to be placed in spaces with adequate topological structure.

We denote by $\mathscr{K}(X)$ the set of compact subsets of $X$ and by $\mathscr{F}(X)$ the set of fuzzy sets with compact $\alpha$-levels defined over $X$, where $X$ has some metric structure. Mustafa and Sims [8] introduced the definition of a generalized metric space, briefly, $G$-metric spaces. In [9] the $G$-metric is introduced in $\mathscr{K}(X)$ and, in [10], the similar construction is made to establish the $G$-metric in the set $\mathscr{F}(X)$. In both cases the structure of the basic $G$-metric space [3-5] is used to define the Hausdorff $G$-metric by the metric $d_{G}$ derived from $G$ metric $G$. In our paper the basic space is the metric space (instead of $G$-metric space) and, using the same idea as in $[9,10]$, the Hausdorff $G$-metric $\mathscr{G}$ is introduced, but metric $d$ of the original metric space $(X, d)$ is used instead of the derived $d_{G}$ metric. In spite of the fact that the relation of the basic and the derived spaces is simpler than in [10] the structure of generalized metric space $(\mathscr{F}(X), \mathscr{G})$ is not reduced. Further, we analyse the existence and uniqueness of a common fixed point for the family $\left\{f_{i}\right\}$ of self-mappings in the set of fuzzy sets $\mathscr{F}(X)$ endowed with generalized metric $\mathscr{G}$. The different type of generalized contractive condition is considered using a nondecreasing, right continuous function 
$\Phi:[0, \infty) \rightarrow[0, \infty)$. Putting different additional conditions on $\Phi$, we can follow how $\Phi$ influences the other conditions, related technique of proving, and the final fixed point result.

For more fixed point results for mappings defined in $G$ metric spaces of fuzzy sets, we refer the reader to [10-12].

\section{Preliminaries}

Definition 1 (see [8]). Let $X$ be a nonempty set, and let $G$ : $X \times X \times X \rightarrow \mathbb{R}^{+}$be a function satisfying the following properties:

(G1) $G(x, y, z)=0$ if $x=y=z$;

(G2) $0<G(x, x, y)$, for all $x, y \in X$, with $x \neq y$;

(G3) $G(x, x, y) \leq G(x, y, z)$, for all $x, y, z \in X$, with $z \neq y$;

(G4) $G(x, y, z)=G(x, z, y)=G(y, z, x)=\cdots$, (symmetry in all three variables);

(G5) $G(x, y, z) \leq G(x, a, a)+G(a, y, z)$, for all $x, y, z, a \in$ $X$.

Then function $G$ is called a generalized metric, abbreviated $G$ metric, on $X$ and the pair $(X, G)$ is called a $G$-metric space. If $G(x, y, y)=G(y, x, x)$ for all $x, y \in X$, then $(X, G)$ is symmetric.

Clearly, these properties are satisfied when $G(x, y, z)$ is the perimeter of the triangle with vertices at $x, y$, and $z \in \mathbb{R}^{2}$; moreover taking $a$ in the interior of the triangle shows that (G5) is the best possible.

Example 2 (see $[8])$. Let $(X, d)$ be an ordinary metric space; then $(X, d)$ defines $G$-metrics on $X$ by

$$
\begin{gathered}
G_{s}(x, y, z)=d(x, y)+d(y, z)+d(x, z), \\
G_{m}(x, y, z)=\max \{d(x, y), d(y, z), d(x, z)\} .
\end{gathered}
$$

The following useful properties of a $G$-metric are readily derived from the axioms.

Proposition 3 (see [8]). Let $(X, G)$ be a $G$-metric space; then for any $x, y, z$, and a from $X$ it follows that

(1) if $G(x, y, z)=0$, then $x=y=z$,

(2) $G(x, y, z) \leq G(x, x, y)+G(x, x, z)$,

(3) $G(x, y, y) \leq 2 G(y, x, x)$,

(4) $G(x, y, z) \leq G(x, a, z)+G(a, y, z)$,

(5) $G(x, y, z) \leq(2 / 3)(G(x, y, a)+G(x, a, z)+G(a, y, z))$,

(6) $G(x, y, z) \leq G(x, a, a)+G(y, a, a)+G(z, a, a)$.

Definition 4 (see [8]). Let $(X, G)$ be a $G$-metric space; and let $\left\{x_{n}\right\}$ be a sequence of points of $X$. A point $x \in X$ is said to be the limit of the sequence $\left\{x_{n}\right\}$ if $\lim _{n, m \rightarrow \infty} G\left(x, x_{n}, x_{m}\right)=0$, and one says that the sequence $\left\{x_{n}\right\}$ is $G$-convergent to $x$.

Proposition 5 (see $[8])$. Let $(X, G)$ be a $G$-metric space, then for a sequence $\left\{x_{n}\right\} \subseteq X$ and a point $x \in X$ the following are equivalent:
(1) $\left\{x_{n}\right\}$ is G-convergent to $x$,

(2) $G\left(x_{n}, x_{n}, x\right) \rightarrow 0$ as $n \rightarrow \infty$,

(3) $G\left(x_{n}, x, x\right) \rightarrow 0$ as $n \rightarrow \infty$.

Definition 6 (see [8]). Let $(X, G)$ be a $G$-metric space; a sequence $\left\{x_{n}\right\}$ is called $G$-Cauchy if, for every $\epsilon>0$, there is $N \in \mathbb{N}$ such that $G\left(x_{n}, x_{m}, x_{l}\right)<\epsilon$, for all $n, m, l \geq N$, that is, if $G\left(x_{n}, x_{m}, x_{l}\right) \rightarrow 0$ as $n, m, l \rightarrow \infty$.

Proposition 7 (see [8]). In a $G$-metric space $(X, G)$, the following are equivalent:

(1) the sequence $\left\{x_{n}\right\}$ is G-Cauchy,

(2) for every $\varepsilon>0$, there exists an $n_{0} \in \mathbb{N}$ such that $G\left(x_{n}\right.$, $\left.x_{m}, x_{m}\right)<\varepsilon$, for all $n, m \geq n_{0}$.

A $G$-metric space $(X, G)$ is $G$-complete (or complete $G$ metric) if every $G$-Cauchy sequence in $(X, G)$ is $G$-convergent in $(X, G)$.

Proposition 8 (see [8]). Every G-metric space $(X, G)$ defines a metric space $\left(X, d_{G}\right)$ by

$$
d_{G}(x, y)=G(x, y, y)+G(y, x, x), \quad \forall x, y \in X .
$$

Note that if $(X, G)$ is a symmetric $G$-metric space, then

$$
d_{G}(x, y)=2 G(x, y, y), \quad \forall x, y \in X .
$$

However, if $(X, G)$ is nonsymmetric, then by $G$-metric properties

$$
\frac{3}{2} G(x, y, y) \leq d_{G}(x, y) \leq 3 G(x, y, y), \quad \forall x, y \in X,
$$

and in general these inequalities cannot be improved.

Proposition 9 (see [8]). Let $(X, G)$ be a $G$-metric space; then the function $G(x, y, z)$ is jointly continuous in all three of its variables.

In [13] it was shown that if $(X, G)$ is a $G$-metric space, putting $\varrho(x, y)=G(x, y, y),(X, \varrho)$ is a quasi-metric space (generally, $\varrho$ is not symmetric). It is well known that any quasi-metric induces different metrics and mostly used are

$$
\begin{aligned}
& \text { (u) } \mu(x, y)=\varrho(x, y)+\varrho(y, x), \\
& (\rho) \rho(x, y)=\max \{\varrho(x, y), \varrho(y, x)\} .
\end{aligned}
$$

The following result is an immediate consequence of the above definitions and relations.

Theorem 10. Let $(X, G)$ be a $G$-metric space and let $D \in$ $\{\mu, \rho\}$. Then

(1) $\left\{x_{n}\right\} \subset X$ is G-convergent to $x \in X$ if and only if $\left\{x_{n}\right\}$ is convergent to $x$ in $(X, D)$;

(2) $\left\{x_{n}\right\} \subset X$ is G-Cauchy if and only if $\left\{x_{n}\right\}$ is Cauchy in $(X, D)$;

(3) $(X, G)$ is $G$-complete if and only if $(X, D)$ is complete. 
Recently, Samet et al. [14] and Jleli and Samet [13] observed that some fixed point theorems in context of $G$-metric space can be proved (by simple transformation) using related existing results in the setting of (quasi) metric space. Namely, if the contraction condition of the fixed point theorem on $G$-metric space can be reduced to two variables, then one can construct an equivalent fixed point theorem in setting of usual metric space. This idea is not completely new, but it was not successfully used before; see [15]. Karapinar and Agarval in [16] continued to develop Jleli-Samet technique in $G$-metric space, but, on the other side, they proved fixed point theorems on the context of $G$-metric space for which Jleli-Samet technique is not applicable. So, in some cases, as it is noticed even in Jleli and Samet paper [13], when the contraction condition is of nonlinear type, this strategy cannot be always successfully used. This is exactly the case in our paper where in fixed point results the use of JleliSamet technique does not give satisfactory results. If, for instance, the function $f$ in (17) is not independent of the variable $\mu \in \mathscr{F}(X)$, then $n(\mu), \mu \in \mathscr{F}(X)$, the exponent factor in contraction conditions in our theorems, is not the constant function (as it is the case in our paper), implying that conditions which the contractor $\Phi$ in related metric space must satisfy become significantly more restrictive if the JleliSamet technique is used. But, using directly $G$-metric $G$, the proofs of theorems in our paper are given. The conclusion is that results from our paper cannot be deduced from the usual one in metric or quasi-metric space and cannot be derived from the results of Samet et al. [14] and Jleli and Samet [13].

For some fixed point results in $G$-metric spaces we refer to $[17-22]$.

\section{Fuzzy Generalized Metric Space}

Let $(X, d)$ be a metric space and let $\mathscr{K}(X)$ be the set of all nonempty compact subsets of $X$. For all $x \in X$ and all $A, B \in \mathscr{K}(X)$, let $d(x, A)=\inf _{a \in A} d(x, a)$ and $d(A, B)=$ $\inf _{a \in A, b \in B} d(a, b)$. The Hausdorff metric on $\mathscr{K}(X)$ is defined by $H(A, B)=\max \left\{\sup _{a \in A} d(a, B), \sup _{b \in B} d(b, A)\right\}$. By $\mathscr{F}(X)$ we denote the set of all fuzzy sets $\mu: X \rightarrow[0,1]$ with compact $\alpha$-levels $\mu_{\alpha}, \alpha \in(0,1]$, where $\mu_{\alpha}=\{x \in X: \mu(x) \geq$ $\alpha\}$ and bounded support $\mu_{0}=\overline{\mathrm{U}_{\alpha \in(0,1]} \mu_{\alpha}}$. In the set $\mathscr{F}(X)$ the metric is introduced by $\delta(\mu, \nu)=\sup _{\alpha \in(0,1]} H\left(\mu_{\alpha}, \nu_{\alpha}\right)$. If $\mu, \nu \in \mathscr{F}(X)$, then $(\mu \subseteq \nu) \Leftrightarrow(\mu(x) \leq \nu(x))$ for all $x \in X$ and $(\mu=\nu) \Leftrightarrow(\mu \subseteq \nu \wedge \nu \subseteq \mu)$. by

The function $\mathscr{G}: \mathscr{F}(X) \times \mathscr{F}(X) \times \mathscr{F}(X) \rightarrow \mathbb{R}^{+}$is defined

$$
\mathscr{G}(\mu, \nu, \eta)=\max \{\mathscr{L}(\mu, \nu, \eta), \mathscr{L}(\eta, \mu, \nu), \mathscr{L}(\nu, \eta, \mu)\},
$$

where

$$
\begin{aligned}
& \mathscr{L}(\mu, v, \eta) \\
& \quad=\sup _{\alpha \in(0,1]}\left\{\sup _{x \in \mu_{\alpha}}\left\{d\left(x, v_{\alpha}\right)+d\left(v_{\alpha}, \eta_{\alpha}\right)+d\left(x, \eta_{\alpha}\right)\right\}\right\} .
\end{aligned}
$$

Lemma 11. For all $\mu, \nu \in \mathscr{F}(X), \delta(\mu, \nu)<\mathscr{G}(\mu, \nu, \nu) \leq$ $2 \delta(\mu, \nu)$.

Proof. Since

$$
\begin{gathered}
\mathscr{L}(\mu, \nu, \nu)=\sup _{\alpha \in(0,1]}\left\{2 \sup _{x \in \mu_{\alpha}} d\left(x, v_{\alpha}\right)\right\}, \\
\mathscr{L}(\nu, \mu, \nu)=\mathscr{L}(\nu, \nu, \mu) \\
=\sup _{\alpha \in(0,1]}\left\{\sup _{y \in \nu_{\alpha}} d\left(y, \mu_{\alpha}\right)+d\left(\mu_{\alpha}, \nu_{\alpha}\right)\right\},
\end{gathered}
$$

from (5) and (6), we obtain

$$
\begin{gathered}
\mathscr{G}(\mu, v, \nu) \\
=\max \left\{\sup _{\alpha \in(0,1]}\left\{2 \sup _{x \in \mu_{\alpha}} d\left(x, v_{\alpha}\right)\right\},\right. \\
\left.\sup _{\alpha \in(0,1]}\left\{\sup _{y \in v_{\alpha}} d\left(y, \mu_{\alpha}\right)+d\left(\mu_{\alpha}, v_{\alpha}\right)\right\}\right\} \\
=\sup _{\alpha \in(0,1]}\left\{\operatorname { m a x } \left\{2 \sup _{x \in \mu_{\alpha}} d\left(x, v_{\alpha}\right), \sup _{y \in \nu_{\alpha}} d\left(y, \mu_{\alpha}\right)\right.\right. \\
\left.\left.+d\left(\mu_{\alpha}, v_{\alpha}\right)\right\}\right\} .
\end{gathered}
$$

The next two inequalities,

$$
\begin{gathered}
\mathscr{G}(\mu, v, v) \\
=\sup _{\alpha \in(0,1]}\left\{\operatorname { m a x } \left\{2 \sup _{x \in \mu_{\alpha}} d\left(x, v_{\alpha}\right), \sup _{y \in \nu_{\alpha}} d\left(y, \mu_{\alpha}\right)\right.\right. \\
\left.\left.+d\left(\mu_{\alpha}, v_{\alpha}\right)\right\}\right\} \\
\leq 2 \sup _{\alpha \in(0,1]}\left\{\max \left\{\sup _{x \in \mu_{\alpha}} d\left(x, v_{\alpha}\right), \sup _{y \in \nu_{\alpha}} d\left(y, \mu_{\alpha}\right)\right\}\right\} \\
=2 \sup _{\alpha \in(0,1]}\left\{H\left(\mu_{\alpha}, v_{\alpha}\right)\right\}=2 \delta(\mu, \nu), \\
\mathscr{G}(\mu, \nu, v) \\
=\sup _{\alpha \in(0,1]}\left\{\operatorname { m a x } \left\{2 \sup _{x \in \mu_{\alpha}} d\left(x, v_{\alpha}\right), \sup _{y \in \nu_{\alpha}} d\left(y, \mu_{\alpha}\right)\right.\right. \\
\left.\left.\quad+d\left(\mu_{\alpha}, v_{\alpha}\right)\right\}\right\} \\
>\sup _{\alpha \in(0,1]}\left\{\max \left\{\sup _{x \in \mu_{\alpha}} d\left(x, v_{\alpha}\right), \sup _{y \in v_{\alpha}} d\left(y, \mu_{\alpha}\right)\right\}\right\} \\
=\sup _{\alpha \in(0,1]}\left\{H\left(\mu_{\alpha}, v_{\alpha}\right)\right\}=\delta(\mu, v),
\end{gathered}
$$

imply the relation $\delta(\mu, \nu)<\mathscr{G}(\mu, \nu, \nu) \leq 2 \delta(\mu, \nu)$, what we had to prove. 
Proposition 12. If $(X, d)$ is a complete metric space, then $(\mathscr{F}(X), \mathscr{G})$ is a complete $G$-metric fuzzy space.

Proof. Properties (G1), (G2), and (G4) from Definition 1 are obvious, so the proof is omitted.

(G3) Since $d(x, A) \leq d(x, B)+d(A, B)$ for all $x \in X$ and all $A, B \in \mathscr{K}(X)$, we have

$$
\begin{aligned}
& \mathscr{L}(\mu, \nu, v) \\
& =\sup _{\alpha \in(0,1]}\left\{\sup _{x \in \mu_{\alpha}}\left\{d\left(x, v_{\alpha}\right)+d\left(x, v_{\alpha}\right)+d\left(\nu_{\alpha}, v_{\alpha}\right)\right\}\right\} \\
& \leq \sup _{\alpha \in(0,1]}\left\{\sup _{x \in \mu_{\alpha}}\left\{d\left(x, v_{\alpha}\right)+d\left(x, \eta_{\alpha}\right)+d\left(\eta_{\alpha}, \nu_{\alpha}\right)\right\}\right\} \\
& =\mathscr{L}(\mu, \nu, \eta) .
\end{aligned}
$$

By the same way we show that

$$
\mathscr{L}(\nu, \mu, \nu) \leq \mathscr{L}(\eta, \mu, \nu), \quad \mathscr{L}(\nu, \nu, \mu) \leq \mathscr{L}(\nu, \eta, \mu),
$$

which implies that $\mathscr{G}(\mu, v, \nu) \leq \mathscr{G}(\mu, v, \eta)$.

(G5) To prove that $\mathscr{G}(\mu, \nu, \eta) \leq \mathscr{G}(\mu, \theta, \theta)+\mathscr{G}(\theta, \nu, \eta)$, we consider the related inequality for $\mathscr{L}$ :

$$
\begin{aligned}
& \mathscr{L}(\mu, v, \eta) \\
& =\sup _{\alpha \in(0,1]}\left\{\sup _{x \in \mu_{\alpha}}\left\{d\left(x, v_{\alpha}\right)+d\left(x, \eta_{\alpha}\right)+d\left(\eta_{\alpha}, v_{\alpha}\right)\right\}\right\} \\
& \leq \sup _{\alpha \in(0,1]}\left\{\operatorname { s u p } _ { x \in \mu _ { \alpha } } \left\{d\left(x, \theta_{\alpha}\right)+d\left(\theta_{\alpha}, v_{\alpha}\right)+d\left(x, \theta_{\alpha}\right)\right.\right. \\
& \left.\left.+d\left(\theta_{\alpha}, \eta_{\alpha}\right)+d\left(\eta_{\alpha}, v_{\alpha}\right)\right\}\right\} \\
& \leq \sup _{\alpha \in(0,1]}\left\{\operatorname { s u p } _ { x \in \mu _ { \alpha } } \left\{d\left(x, \theta_{\alpha}\right)+\sup _{t \in \theta_{\alpha}} d\left(t, v_{\alpha}\right)+d\left(x, \theta_{\alpha}\right)\right.\right. \\
& \left.\left.\quad+\sup _{t \in \theta_{\alpha}}\left(t, \eta_{\alpha}\right)+d\left(\eta_{\alpha}, v_{\alpha}\right)\right\}\right\} \\
& =\sup _{\alpha \in(0,1]}\left\{\sup _{x \in \mu_{\alpha}}\left\{d\left(x, \theta_{\alpha}\right)+d\left(x, \theta_{\alpha}\right)+d\left(\theta_{\alpha}, \theta_{\alpha}\right)\right\}\right. \\
& \left.+\sup _{t \in \theta_{\alpha}}\left\{d\left(t, v_{\alpha}\right)+d\left(t, \eta_{\alpha}\right)+d\left(\eta_{\alpha}, v_{\alpha}\right)\right\}\right\}
\end{aligned}
$$

Analogously, $\mathscr{L}(\eta, \mu, \nu) \leq \mathscr{L}(\theta, \mu, \theta)+\mathscr{L}(\eta, \theta, \nu)$ and $\mathscr{L}(\nu$, $\eta, \mu) \leq \mathscr{L}(\theta, \theta, \mu)+\mathscr{L}(\nu, \eta, \theta)$. All three inequalities together imply that $\mathscr{G}(\mu, \nu, \eta) \leq \mathscr{G}(\mu, \theta, \theta)+\mathscr{G}(\theta, \nu, \eta)$.

The completeness of $(\mathscr{F}(X), \mathscr{G})$ is a consequence of the completeness of $(\mathscr{F}(X), \delta)$ and inequality from the previous lemma.
Proposition 13. G-metric fuzzy space $(\mathscr{F}(X), \mathscr{G})$ is not symmetric.

Proof. We prove that $\mathscr{G}(\mu, \nu, \nu) \neq \mathscr{G}(\nu, \mu, \mu)$. If

$$
\begin{gathered}
\sup _{\alpha \in(0,1]} \sup _{x \in \mu_{\alpha}} d\left(x, v_{\alpha}\right)=A, \\
\sup _{\alpha \in(0,1]} \sup _{y \in \nu_{\alpha}} d\left(y, \mu_{\alpha}\right)=b, \\
\sup _{\alpha \in(0,1]} d\left(\nu_{\alpha}, \mu_{\alpha}\right)=C,
\end{gathered}
$$

then

$$
\begin{aligned}
\mathscr{G}(\mu, \nu, \nu) & =\max \{2 A, B+C\} \\
& \neq \mathscr{G}(\nu, \mu, \mu) \\
& =\max \{2 B, A+C\} .
\end{aligned}
$$

\section{Fixed Point for Contractive Iterate at the Point and Orbitally Contractive at the Point Fuzzy Mappings}

A generalization of the contraction principle can be obtained using different type of a nondecreasing right continuous function $\Phi:[0, \infty) \rightarrow[0, \infty)$. The most usual additional properties imposed on $\Phi$ are given using a combination of the next seven conditions:

$\left(\phi_{1}\right) \Phi(0)=0$,

$\left(\phi_{2}\right) \Phi(x)<x$, for all $x>0$,

$\left(\phi_{3}\right) \lim _{i \rightarrow \infty} \Phi^{i}(x)=0$, for all $x>0$,

$\left(\phi_{4}\right)\left\{x_{i}\right\} \subset[0, \infty)$ is a sequence such that $x_{i+1} \leq \Phi\left(x_{i}\right) ;$ then $\lim _{i \rightarrow \infty} x_{i}=0$.

$\left(\phi_{5}\right)$ for any $x \geq 0$ there exists a $y(x) \geq 0, y(x)=$ $\sup _{y \geq 0}\{y \leq x+\Phi(y)\}$

$\left(\phi_{6}\right) \lim _{x \rightarrow \infty}(x-\Phi(x))=\infty$,

$\left(\phi_{7}\right) \sum_{i=1}^{\infty} \Phi^{i}(x)<\infty$, for all $x>0$.

Some of the noted properties of $\Phi$ are equivalent, some of them imply other, and some of them are incompatible. The next lemma discusses some of the relations between properties $\left(\phi_{1}\right)-\left(\phi_{7}\right)$, especially those which are used in this paper to define a generalized contraction.

Lemma 14. Let $\Phi:[0, \infty) \rightarrow[0, \infty)$ be a nondecreasing right continuous function. Then

(i) $\left(\phi_{2}\right) \Leftrightarrow\left(\phi_{3}\right) \Leftrightarrow\left(\phi_{4}\right)$,

(ii) $\left(\phi_{7}\right) \Rightarrow\left(\phi_{k}\right) \Rightarrow\left(\phi_{1}\right)$, where $k \in\{2,3,4\}$,

(iii) $\left(\phi_{5}\right)+\left(\phi_{2}\right) \nRightarrow\left(\phi_{7}\right)$ and $\left(\phi_{6}\right)+\left(\phi_{2}\right) \nRightarrow\left(\phi_{7}\right)$,

(iv) $\left(\phi_{7}\right) \nRightarrow\left(\phi_{5}\right)$ and $\left(\phi_{7}\right) \nRightarrow\left(\phi_{6}\right)$. 
Proof. (i) It is enough to prove that $\left(\phi_{4}\right) \Rightarrow\left(\phi_{2}\right) \Leftrightarrow\left(\phi_{3}\right) \Rightarrow$ $\left(\phi_{4}\right)$.

$\left(\phi_{2}\right) \Rightarrow\left(\phi_{3}\right)$. We assume that for some $x>0, \lim _{i \rightarrow \infty} \Phi^{i}(x)=$ $a>0$. Since $\left\{\Phi^{i}(x)\right\}$ is nonincreasing sequence, by the right continuity of $\Phi, \Phi(a)=\Phi \lim _{i \rightarrow \infty} \Phi^{i}(x)=$ $\lim _{i \rightarrow \infty} \Phi^{i+1}(x)=a>0$; that is, $0<a=\Phi(a)$ which contradicts $\left(\phi_{2}\right)$.

$\left(\phi_{3}\right) \Rightarrow\left(\phi_{2}\right)$. If for some $x>0, \Phi(x) \geq x$, then, knowing that $\Phi$ is nondecreasing, $\Phi^{i}(x) \geq \Phi^{i-1}(x) \geq \cdots \geq \Phi(x) \geq x>0$. It means that $\lim _{i \rightarrow \infty} \Phi^{i}(x) \neq 0$, which contradicts $\left(\phi_{3}\right)$.

$\left(\phi_{3}\right) \Rightarrow\left(\phi_{4}\right)$. Let $\left\{x_{i}\right\} \subset[0, \infty)$ be any sequence such that $x_{i+1} \leq \Phi\left(x_{i}\right)$. Then $x_{i} \leq \Phi\left(x_{i-1}\right) \leq \Phi^{2}\left(x_{i-2}\right) \leq \cdots \leq \Phi^{i}\left(x_{0}\right)$ and $\lim _{i \rightarrow \infty} x_{i} \leq \lim _{i \rightarrow \infty} \Phi^{i}\left(x_{0}\right)=0$.

$\left(\phi_{4}\right) \Rightarrow\left(\phi_{2}\right)$. If $\left(\phi_{2}\right)$ does not hold, that is, there exists a $y>0$, $y \leq \Phi(y)$, then putting $x_{i}=y$, for all $i=0,1, \ldots$, we have the sequence with $y=x_{i+1} \leq \Phi(y)=\Phi\left(x_{i}\right)$, but that sequence does not converge to 0 .

(ii) is obvious, so the proof is omitted.

(iii) Function

$$
\Phi(x)= \begin{cases}(n+2)^{-1}, & (n+1)^{-1} \leq x<n^{-1}, n \in \mathbb{N}, \\ 3^{-1}, & 1 \leq x\end{cases}
$$

satisfies $\left(\phi_{5}\right),\left(\phi_{6}\right)$ and $\left(\phi_{2}\right)$ but not $\left(\phi_{7}\right)$.

(iv) Function

$$
\Phi(x)= \begin{cases}\left(\frac{x}{2}\right)^{2}, & 0 \leq x<2 \\ x-1, & 2 \leq x\end{cases}
$$

satisfies $\left(\phi_{7}\right)$ but not $\left(\phi_{5}\right)$ nor $\left(\phi_{6}\right)$.

Theorem 15. Let $(X, d)$ be a complete metric space, let $(\mathscr{F}(X), \mathscr{G})$ be related $G$-metric fuzzy space, and let $\mathscr{B} \subseteq \mathscr{F}(X)$. Further, let $\left\{f_{i}\right\}$ be the sequence of self-mappings of $\mathscr{F}(X)$ such that, for all $i \in \mathbb{N}, f_{i}(\mathscr{B}) \subseteq \mathscr{B}$ and for each $\mu \in \mathscr{F}(X)$ there exists an $n(\mu) \in \mathbb{N}$ such that

$$
\begin{gathered}
\mathscr{G}\left(f_{i}^{n(\mu)}(\nu), f_{j}^{n(\mu)}(\mu), f_{j}^{n(\mu)}(\mu)\right) \\
\leq \Phi(\max \{\mathscr{G}(\nu, \mu, \mu), \\
2^{-1}\left[\mathscr{G}\left(\nu, f_{j}^{n(\mu)}(\mu), f_{j}^{n(\mu)}(\mu)\right)\right. \\
\left.+\mathscr{G}\left(\mu, f_{j}^{n(\mu)}(\mu), f_{j}^{n(\mu)}(\mu)\right)\right], \\
2^{-1}\left[\mathscr{G}\left(\nu, f_{j}^{n(\mu)}(\mu), f_{j}^{n(\mu)}(\mu)\right)\right. \\
\left.\left.\left.+\mathscr{G}\left(f_{i}^{n(\mu)}(\nu), \mu, \mu\right)\right]\right\}\right),
\end{gathered}
$$

for all $i, j \in \mathbb{N}$ and all $\nu \in \mathscr{B}$, where $\Phi$ satisfies $\left(\phi_{2}\right)$. If there exists $\mu^{*} \in \mathscr{B}$ such that $f_{i}^{n\left(\mu^{*}\right)}\left(\mu^{*}\right)=\mu^{*}$ for all $i \in \mathbb{N}$, then $\mu^{*}$ is a unique common fixed point for $\left\{f_{i}\right\}$ in $\mathscr{B}$ and, for every $\mu_{1} \in \mathscr{F}(X)$, the sequence $\mu_{j+1}=f_{i}^{n\left(\mu^{*}\right)}\left(\mu_{j}\right), i \in \mathbb{N}$, converges to $\mu^{*}$.
Proof. First we prove that $\mu^{*}$ is a unique point in $\mathscr{B}$ with the property that $f_{i}^{n\left(\mu^{*}\right)}\left(\mu^{*}\right)=\mu^{*}, i \in \mathbb{N}$. If $\nu \in \mathscr{B}, \nu \neq \mu^{*}$, $f_{i}^{n\left(\mu^{*}\right)}(\nu)=\nu, i \in \mathbb{N}$, then

$$
\begin{aligned}
\mathscr{G}\left(\nu, \mu^{*}, \mu^{*}\right) & \leq \mathscr{G}\left(f_{i}^{n\left(\mu^{*}\right)}(\nu), f_{j}^{n\left(\mu^{*}\right)}\left(\mu^{*}\right), f_{j}^{n\left(\mu^{*}\right)}\left(\mu^{*}\right)\right) \\
& \leq \Phi\left(\mathscr{G}\left(\nu, \mu^{*}, \mu^{*}\right)\right) .
\end{aligned}
$$

By the property $\Phi(t)<t, t>0$, since $\mathscr{G}\left(\nu, \mu^{*}, \mu^{*}\right)>0$, we have the contradiction; that is, the assumption $\mu^{*} \neq \nu$ is not correct.

Further, since

$$
f_{i}\left(\mu^{*}\right)=f_{i}\left(f_{i}^{n\left(\mu^{*}\right)}\left(\mu^{*}\right)\right)=f_{i}^{n\left(\mu^{*}\right)+1}\left(\mu^{*}\right)=f_{i}^{n\left(\mu^{*}\right)}\left(f_{i}\left(\mu^{*}\right)\right),
$$

it follows that $f_{i}\left(\mu^{*}\right)=\mu^{*}$ for all $i \in \mathbb{N}$.

Now, for some $\mu_{1} \in \mathscr{B}$, we form the sequence $\mu_{i+1}=$ $f_{i}^{n\left(\mu^{*}\right)}\left(\mu_{i}\right)$.

If $\mu_{1}=\mu^{*}$, then $\mu_{i}=f_{i-1}^{n\left(\mu^{*}\right)}\left(f_{i-2}^{n\left(\mu^{*}\right)} \cdots\left(f_{1}^{n\left(\mu^{*}\right)}\left(\mu^{*}\right)\right) \cdots\right)=$ $\mu^{*}$ and the sequence $\left\{\mu_{i}\right\}$ converges to $\mu^{*}$.

If $\mu_{i} \neq \mu^{*}$, in order to prove that the sequence $\left\{\mu_{i}\right\}$ converges to $\mu^{*}$, we consider the sequence $\mathscr{G}\left(\mu_{i+1}, \mu^{*}, \mu^{*}\right)$, $i \in \mathbb{N}$ :

$$
\begin{aligned}
& \mathscr{G}\left(\mu_{i+1}, \mu^{*}, \mu^{*}\right) \\
& =\mathscr{G}\left(f_{i}^{n\left(\mu^{*}\right)}\left(\mu_{i}\right), f_{j}^{n\left(\mu^{*}\right)}\left(\mu^{*}\right), f_{j}^{n\left(\mu^{*}\right)}\left(\mu^{*}\right)\right) \\
& \leq \Phi\left(\operatorname { m a x } \left\{\mathscr{G}\left(\mu_{i}, \mu^{*}, \mu^{*}\right),\right.\right. \\
& 2^{-1}\left[\mathscr{G}\left(\mu_{i}, \mu^{*}, \mu^{*}\right)+\mathscr{G}\left(\mu^{*}, \mu^{*}, \mu^{*}\right)\right], \\
& \left.\left.2^{-1}\left[\mathscr{G}\left(\mu_{i}, \mu^{*}, \mu^{*}\right)+\mathscr{G}\left(\mu_{i+1}, \mu^{*}, \mu^{*}\right)\right]\right\}\right) \\
& =\Phi\left(\operatorname { m a x } \left\{\mathscr{G}\left(\mu_{i}, \mu^{*}, \mu^{*}\right),\right.\right. \\
& \left.\left.2^{-1}\left[\mathscr{G}\left(\mu_{i}, \mu^{*}, \mu^{*}\right)+\mathscr{G}\left(\mu_{i+1}, \mu^{*}, \mu^{*}\right)\right]\right\}\right) .
\end{aligned}
$$

If we choose the option that

$$
\begin{aligned}
\max & \left\{\mathscr{G}\left(\mu_{i}, \mu^{*}, \mu^{*}\right), 2^{-1}\left[\mathscr{G}\left(\mu_{i}, \mu^{*}, \mu^{*}\right)+\mathscr{G}\left(\mu_{i+1}, \mu^{*}, \mu^{*}\right)\right]\right\} \\
& =2^{-1}\left[\mathscr{G}\left(\mu_{i}, \mu^{*}, \mu^{*}\right)+\mathscr{G}\left(\mu_{i+1}, \mu^{*}, \mu^{*}\right)\right],
\end{aligned}
$$

it implies that

$$
\mathscr{G}\left(\mu_{i}, \mu^{*}, \mu^{*}\right) \leq \mathscr{G}\left(\mu_{i+1}, \mu^{*}, \mu^{*}\right) .
$$

On the other hand, in that case

$$
\begin{aligned}
\mathscr{G}\left(\mu_{i+1}, \mu^{*}, \mu^{*}\right) & \leq \Phi\left(2^{-1}\left[\mathscr{G}\left(\mu_{i}, \mu^{*}, \mu^{*}\right)+\mathscr{G}\left(\mu_{i+1}, \mu^{*}, \mu^{*}\right)\right]\right) \\
& <2^{-1} \mathscr{G}\left(\mu_{i}, \mu^{*}, \mu^{*}\right)+2^{-1} \mathscr{G}\left(\mu_{i+1}, \mu^{*}, \mu^{*}\right)
\end{aligned}
$$

that is

$$
\mathscr{G}\left(\mu_{i+1}, \mu^{*}, \mu^{*}\right)<\mathscr{G}\left(\mu_{i}, \mu^{*}, \mu^{*}\right) .
$$


It is obvious that (22) contradicts (24). So,

$$
\mathscr{G}\left(\mu_{i+1}, \mu^{*}, \mu^{*}\right) \leq \Phi\left(\mathscr{G}\left(\mu_{i}, \mu^{*}, \mu^{*}\right)\right) .
$$

Now, applying that procedure $i$ times and letting $i \rightarrow \infty$, we get

$$
\begin{aligned}
\mathscr{G}\left(\mu_{i+1}, \mu^{*}, \mu^{*}\right) & \leq \Phi\left(\mathscr{G}\left(\mu_{i}, \mu^{*}, \mu^{*}\right)\right) \\
& \leq \cdots \leq \Phi^{i}\left(\mathscr{G}\left(\mu_{1}, \mu^{*}, \mu^{*}\right)\right) .
\end{aligned}
$$

Since $\mu_{1} \neq \mu^{*}, \mathscr{G}\left(\mu_{i}, \mu^{*}, \mu^{*}\right)>0$ and $\lim _{i \rightarrow \infty} \mathscr{G}\left(\mu_{i+1}, \mu^{*}, \mu^{*}\right)=$ 0 . The last relation proves that the sequence $\left\{\mu_{i}\right\}$ converges to $\mu^{*}$.

Theorem 16. Let $(X, d)$ be a complete metric space, $f$ : $\mathscr{F}(X) \rightarrow \mathscr{F}(X)$, where $(\mathscr{F}(X), \mathscr{G})$ is related $G$-metric fuzzy space, and let $\Phi:[0, \infty) \rightarrow[0, \infty)$ be a subadditive mapping satisfying $\left(\phi_{7}\right)$. If for some $\mu_{0} \in \mathscr{F}(X)$ the orbit $\mathcal{O}\left(f ; \mu_{0}\right)$ is complete and for each $\mu \in \mathcal{O}\left(f ; \mu_{0}\right)$ there exists an $n(\mu) \in \mathbb{N}$ such that

$$
\mathscr{G}\left(f^{n(\mu)}(\nu), f^{n(\mu)}(\mu), f^{n(\mu)}(\mu)\right) \leq \Phi(\mathscr{G}(\nu, \mu, \mu))
$$

for all $\nu \in \mathcal{O}\left(f ; \mu_{0}\right)$, then the sequence $\mu_{i+1}=f^{n\left(\mu_{i}\right)}\left(\mu_{i}\right), i \in \mathbb{N}_{0}$, converges to some $\mu^{*} \in \mathscr{F}(X)$.

If inequality (27) holds for all $\mu \in \overline{\mathcal{O}\left(f ; \mu_{0}\right)}$, then $f^{n\left(\mu^{*}\right)}\left(\mu^{*}\right)=\mu^{*}$ and $f^{i}(\mu) \rightarrow \mu^{*}$ for all $\mu \in \overline{\mathcal{O}\left(f ; \mu_{0}\right)}$. If $f\left(\overline{\left.\mathcal{O}\left(f ; \mu_{0}\right)\right)} \subseteq \overline{\mathcal{O}\left(f ; \mu_{0}\right)}\right.$, then $\mu^{*}$ is the fixed point of $f$.

Proof. First, we show that $\left\{\mu_{i}\right\}_{i \in \mathbb{N}_{0}} \subset \mathscr{F}(X)$ is a Cauchy sequence. For sufficiently large $m \in \mathbb{N}$, there exist $k, r \in \mathbb{N}$, $1 \leq r<n\left(\mu_{0}\right)$ such that $m=k \cdot n\left(\mu_{0}\right)+r$. Using (27), we get

$$
\begin{aligned}
& \mathscr{G}\left(f^{m}\left(\mu_{0}\right), \mu_{0}, \mu_{0}\right) \\
& \leq \mathscr{G}\left(f^{k n\left(\mu_{0}\right)+r}\left(\mu_{0}\right), f^{n\left(\mu_{0}\right)}\left(\mu_{0}\right), f^{n\left(\mu_{0}\right)}\left(\mu_{0}\right)\right) \\
&+\mathscr{G}\left(f^{n\left(\mu_{0}\right)} \mu_{0}, \mu_{0}, \mu_{0}\right) \\
& \leq \Phi\left(\mathscr{G}\left(f^{(k-1) n\left(\mu_{0}\right)+r}\left(\mu_{0}\right), \mu_{0}, \mu_{0}\right)\right) \\
&+\mathscr{G}\left(f^{n\left(\mu_{0}\right)}\left(\mu_{0}\right), \mu_{0}, \mu_{0}\right) \\
& \leq \Phi\left(\mathscr{G}\left(f^{(k-1) n\left(\mu_{0}\right)+r}\left(\mu_{0}\right), f^{n\left(\mu_{0}\right)}\left(\mu_{0}\right), f^{n}\left(\mu_{0}\right)\right)\right. \\
&\left.+\mathscr{G}\left(f^{n\left(\mu_{0}\right)}\left(\mu_{0}\right), \mu_{0}, \mu_{0}\right)\right) \\
&+\mathscr{G}\left(f^{n\left(\mu_{0}\right)}\left(\mu_{0}\right), \mu_{0}, \mu_{0}\right) \\
& \leq \Phi^{2}\left(\mathscr{G}\left(f^{(k-2) n\left(\mu_{0}\right)+r}\left(\mu_{0}\right), \mu_{0}, \mu_{0}\right)\right) \\
&+\Phi\left(\mathscr{G}\left(f^{n\left(\mu_{0}\right)}\left(\mu_{0}\right), \mu_{0}, \mu_{0}\right)\right)+\mathscr{G}\left(f^{n\left(\mu_{0}\right)}\left(\mu_{0}\right), \mu_{0}, \mu_{0}\right) \\
& \leq \cdots \leq \Phi^{k}\left(\mathscr{G}\left(f^{r}\left(\mu_{0}\right), \mu_{0}, \mu_{0}\right)\right) \\
&+\sum_{i=1}^{k-1} \Phi^{i}\left(\mathscr{G}\left(f^{n\left(\mu_{0}\right)}, \mu_{0}, \mu_{0}\right)\right) .
\end{aligned}
$$

Putting $A=\max \left\{\mathscr{G}\left(f^{p}\left(\mu_{0}\right), \mu_{0}, \mu_{0}\right): 1 \leq p \leq n\left(\mu_{0}\right)\right\}$, for all $m \in \mathbb{N}$, the next inequality holds:

$$
\mathscr{G}\left(f^{m}\left(\mu_{0}\right), \mu_{0}, \mu_{0}\right) \leq \sum_{s=1}^{k} \Phi^{s}(A) \leq \sum_{s=1}^{\infty} \Phi^{s}(A)=B<\infty,
$$

and, consequently,

$$
\begin{aligned}
& \mathscr{G}\left(\mu_{m}, \mu_{m}, \mu_{m+1}\right) \\
& =\mathscr{G}\left(f^{n\left(\mu_{m-1}\right)}\left(\mu_{m-1}\right), f^{n\left(\mu_{m-1}\right)}\left(\mu_{m-1}\right),\right. \\
& \left.f^{n\left(\mu_{m}\right)} f^{n\left(\mu_{m-1}\right)}\left(\mu_{m-1}\right)\right) \\
& \leq \Phi\left(\mathscr{G}\left(\mu_{m-1}, \mu_{m-1}, f^{n\left(\mu_{m}\right)}\left(\mu_{m-1}\right)\right)\right) \\
& \leq \cdots \leq \Phi^{m}\left(\mathscr{G}\left(\mu_{0}, \mu_{0}, f^{n\left(\mu_{m}\right)}\left(\mu_{0}\right)\right)\right) \leq \Phi^{m}(B),
\end{aligned}
$$

for all $m \in \mathbb{N}$. Using the last inequality, for every $i, j \in \mathbb{N}$, $i<j$, we have

$$
\begin{aligned}
\mathscr{G}\left(\mu_{i}, \mu_{i}, \mu_{j}\right) \leq & \mathscr{G}\left(\mu_{i}, \mu_{i}, \mu_{i+1}\right) \\
& +\cdots+\mathscr{G}\left(\mu_{k-1}, \mu_{k-1}, \mu_{k}\right) \leq \sum_{s=i}^{j} \Phi^{s}(B),
\end{aligned}
$$

implying that $\left\{\mu_{i}\right\}_{i \in \mathbb{N}}$ is a Cauchy sequence. Since $(\mathscr{F}(X), \mathscr{G})$ is a complete $G$-metric fuzzy space, there exists an $\mu^{*} \in \mathscr{F}(X)$ such that $\lim _{i \rightarrow \infty} \mu_{i}=\mu^{*}$.

In the second part of the theorem, inequality (27) holds for all $\mu \in \overline{\mathcal{O}\left(f ; \mu_{0}\right)}$. Then, the elements $\mu_{i}$ of the sequence $\left\{\mu_{i}\right\}_{i \in \mathbb{N}}$ from the previous part of the proof satisfy the next two relations:

$$
\begin{aligned}
& \mathscr{G}\left(f^{n\left(\mu^{*}\right)}\left(\mu^{*}\right), f^{n\left(\mu^{*}\right)}\left(\mu^{*}\right), f^{n\left(\mu^{*}\right)}\left(\mu_{i}\right)\right) \\
& \leq \Phi\left(\mathscr{G}\left(\mu^{*}, \mu^{*}, \mu_{i}\right)\right), \\
& \mathscr{G}\left(f^{n\left(\mu^{*}\right)}\left(\mu_{i}\right), \mu_{i}, \mu_{i}\right) \\
& =\mathscr{G}\left(f^{n\left(\mu^{*}\right)} f^{n\left(\mu_{i-1}\right)}\left(\mu_{i-1}\right), f^{n\left(\mu_{i-1}\right)}\left(\mu_{i-1}\right),\right. \\
& \left.\quad f^{n\left(\mu_{i-1}\right)}\left(\mu_{i-1}\right)\right) \\
& \leq \Phi\left(\mathscr{G}\left(f^{n\left(\mu^{*}\right)}\left(\mu_{i-1}\right), \mu_{i-1}, \mu_{i-1}\right)\right) \\
& \leq \Phi^{i}\left(\mathscr{G}\left(f^{n\left(\mu^{*}\right)}\left(\mu_{0}\right), \mu_{0}, \mu_{0}\right)\right) .
\end{aligned}
$$

By (32)

$$
\lim _{i \rightarrow \infty} f^{n\left(\mu^{*}\right)}\left(\mu_{i}\right)=f^{n\left(\mu^{*}\right)}\left(\mu^{*}\right)
$$

and by (33)

$$
\lim _{i \rightarrow \infty} \mathscr{G}\left(f^{n\left(\mu^{*}\right)}\left(\mu_{i}\right), \mu_{i}, \mu_{i}\right)=\mathscr{G}\left(f^{n\left(\mu^{*}\right)}\left(\mu^{*}\right), \mu^{*}, \mu^{*}\right)=0 .
$$

Hence, $f^{n\left(\mu^{*}\right)}\left(\mu^{*}\right)=\mu^{*}$. 
To show that $\mu^{*}$ is a unique fixed point of $f^{n\left(\mu^{*}\right)}$ in $\overline{\mathcal{O}\left(f ; \mu_{0}\right)}$, we assume that there exists another point $x^{* *} \in$ $\overline{\mathcal{O}\left(f ; \mu_{0}\right)}$ with the same property. Then

$$
\begin{aligned}
\mathscr{G}\left(x^{* *}, \mu^{*}, \mu^{*}\right) & =\mathscr{G}\left(f^{n\left(\mu^{*}\right)} x^{* *}, f^{n\left(\mu^{*}\right)} \mu^{*}, f^{n\left(\mu^{*}\right)} \mu^{*}\right) \\
& \leq \Phi\left(\mathscr{G}\left(x^{* *}, \mu^{*}, \mu^{*}\right)\right) .
\end{aligned}
$$

That is $x^{* *}=\mu^{*}$. Further, if $f\left(\overline{\left.\mathcal{O}\left(f ; \mu_{0}\right)\right)} \subseteq \overline{\mathcal{O}\left(f ; \mu_{0}\right)}\right.$, then $f \mu^{*}=f\left(f^{n\left(\mu^{*}\right)} \mu^{*}\right)=f^{n\left(\mu^{*}\right)}\left(f \mu^{*}\right)$, implying that $f \mu^{*}=\mu^{*}$.

\section{Subfixed Point for Generalized Contraction Family of Fuzzy Mappings}

We say that $\mu^{*} \in \mathscr{F}(X)$ is a subfixed point of the mapping $f: \mathscr{F}(X) \rightarrow \mathscr{F}(X)$ if and only if $\mu^{*} \subseteq f\left(\mu^{*}\right)$.

The proof of the next two propositions is the same as in [10]; only, instead of the derived metric $d_{G}$, the metric $d$ from original metric space $(X, d)$ is used.

Proposition 17. If $\mu_{1}, \mu_{2}, \nu_{2} \in \mathscr{F}(X)$ and $\mu_{1} \leq \mu_{2}$, then there exists a $v_{1} \in \mathscr{F}(X)$ such that $\mathscr{G}\left(\mu_{1}, v_{1}, v_{1}\right) \leq \mathscr{G}\left(\mu_{2}, v_{2}, v_{2}\right)$.

Proposition 18. If $\mu, \nu, \eta \in \mathscr{F}(X)$ and $\mu \subseteq \nu$, then

(i) $\mathscr{L}(\mu, \eta, \eta) \leq \mathscr{L}(\nu, \eta, \eta)$,

(ii) $\mathscr{L}(\eta, \nu, \nu) \leq \mathscr{L}(\eta, \mu, \mu)$,

(iii) $\mathscr{L}(\mu, \nu, \nu)=0 \Leftrightarrow \mu \subseteq \nu$.

In the next two theorems we consider the existence of a fuzzy set $\mu^{*}$ which represents a common subfixed point for the family of self-mappings $\left\{f_{i}\right\}$, that is, the point such that $\mu^{*} \subseteq f_{i}\left(\mu^{*}\right)$ for all $i \in \mathbb{N}$.

Let $(X, d)$ be a complete metric space, and let $(\mathscr{F}(X), \mathscr{G})$ be related $G$-metric fuzzy space. Further, let for all $\mu, v \in$ $\mathscr{F}(X)$ and all $i, j \in \mathbb{N}, i \neq j$,

$$
\begin{aligned}
\mathscr{G}\left(f_{i}(\nu), f_{j}(\mu), f_{j}(\mu)\right) \\
\leq \Phi\left(\operatorname { m a x } \left\{\left(\mathscr{G}(\nu, \mu, \mu), \mathscr{L}\left(\nu, f_{i}(\nu), f_{i}(\nu)\right),\right.\right.\right. \\
\left.\mathscr{L}\left(\mu, f_{j}(\mu), f_{j}(\mu)\right)\right), \\
\left.\left.\mathscr{L}\left(\nu, f_{j}(\mu), f_{j}(\mu)\right), \mathscr{L}\left(\mu, f_{i}(\nu), f_{i}(\nu)\right)\right\}\right),
\end{aligned}
$$

where $\Phi:[0, \infty) \rightarrow[0, \infty)$.

If $\mu_{0}$ is any element from $\mathscr{F}(X)$ and a $\mu_{1} \in \mathscr{F}(X)$ is chosen such that $\mu_{1} \subseteq f_{1}\left(\mu_{0}\right)$, then, by Proposition 17, there exists a $\mu_{2} \subseteq f_{2}\left(\mu_{1}\right)$, such that

$$
\mathscr{G}\left(\mu_{1}, \mu_{2}, \mu_{2}\right) \leq \mathscr{G}\left(f_{1}\left(\mu_{0}\right), f_{2}\left(\mu_{1}\right), f_{2}\left(\mu_{1}\right)\right) .
$$

By the same principle as for the first three members, the sequence $\left\{\mu_{i}\right\}$ is formed such that

$$
\begin{gathered}
\mu_{i+1} \subseteq f_{i+1}\left(\mu_{i}\right), \quad \forall i \in \mathbb{N}, \\
\mathscr{G}\left(\mu_{i}, \mu_{i+1}, \mu_{i+1}\right) \leq \mathscr{G}\left(f_{i}\left(\mu_{i-1}\right), f_{i+1}\left(\mu_{i}\right), f_{i+1}\left(\mu_{i}\right)\right) .
\end{gathered}
$$

Lemma 19. If $\Phi$ in (37) satisfies $\left(\phi_{5}\right)$ together with $\left(\phi_{2}\right)$ or $\left(\phi_{3}\right)$ or $\left(\phi_{4}\right)$, the sequence $\left\{\mu_{i}\right\}$ defined in (39) is a Cauchy sequence.

Proof. By (37), for any $i, j \in \mathbb{N}$, we have

$$
\begin{gathered}
\mathscr{G}\left(\mu_{i}, \mu_{j}, \mu_{j}\right) \\
\leq \mathscr{G}\left(f_{i}\left(\mu_{i-1}\right), f_{j}\left(\mu_{j-1}\right), f_{j}\left(\mu_{j-1}\right)\right) \\
\leq \Phi\left(\operatorname { m a x } \left\{\mathscr{G}\left(\mu_{i-1}, \mu_{j-1}, \mu_{j-1}\right),\right.\right. \\
\mathscr{L}\left(\mu_{i-1}, f_{i}\left(\mu_{i-1}\right), f_{i}\left(\mu_{i-1}\right)\right), \\
\mathscr{L}\left(\mu_{j-1}, f_{i}\left(\mu_{i-1}\right), f_{i}\left(\mu_{i-1}\right)\right), \\
\mathscr{L}\left(\mu_{j-1}, f_{j}\left(\mu_{j-1}\right), f_{j}\left(\mu_{j-1}\right)\right), \\
\left.\left.\mathscr{L}\left(\mu_{i-1}, f_{j}\left(\mu_{j-1}\right), f_{j}\left(\mu_{j-1}\right)\right)\right\}\right) \\
\leq \Phi\left(\operatorname { m a x } \left\{\mathscr{G}\left(\mu_{i-1}, \mu_{j-1}, \mu_{j-1}\right),\right.\right. \\
\mathscr{L}\left(\mu_{i-1}, f_{i}\left(\mu_{i-1}\right), f_{i}\left(\mu_{i-1}\right)\right), \\
\mathscr{L}\left(\mu_{j-1}, f_{i}\left(\mu_{i-1}\right), f_{i}\left(\mu_{i-1}\right)\right), \\
\mathscr{L}\left(\mu_{j-1}, f_{j}\left(\mu_{j-1}\right), f_{j}\left(\mu_{j-1}\right)\right), \\
\left.\left.\mathscr{L}\left(\mu_{i-1}, f_{j}\left(\mu_{j-1}\right), f_{j}\left(\mu_{j-1}\right)\right)\right\}\right) \\
\mathscr{G}\left(\mu_{j-1}, \mu_{i}, \mu_{i}\right), \mathscr{G}\left(\mu_{j-1}, \mu_{j}, \mu_{j}\right), \\
\left.\left.\mathscr{G}\left(\mu_{i-1}, \mu_{j}, \mu_{j}\right)\right\}\right) . \\
\leq \Phi\left(\operatorname { m a x } \left\{\mathscr{G}\left(\mu_{i-1}, \mu_{j-1}, \mu_{j-1}\right),\right.\right. \\
\mathscr{L}\left(\mu_{i-1}, \mu_{i}, \mu_{i}\right), \mathscr{L}\left(\mu_{j-1}, \mu_{i}, \mu_{i}\right), \\
\left.\left.\mathscr{L}\left(\mu_{j-1}, \mu_{j}, \mu_{j}\right), \mathscr{L}\left(\mu_{i-1}, \mu_{j}, \mu_{j}\right)\right\}\right) \\
\mathscr{G}\left(\mu_{i-1}, \mu_{j-1}, \mu_{j-1}\right), \mathscr{G}\left(\mu_{i-1}, \mu_{i}, \mu_{i}\right), \\
\leq
\end{gathered}
$$

Considering relation (41) for different values $k \leq i, j \leq n$, we get

$$
\sup _{k \leq i, j \leq n}\left\{\mathscr{G}\left(\mu_{i}, \mu_{j}, \mu_{j}\right)\right\} \leq \Phi\left(\sup _{k-1 \leq i, j \leq n}\left\{\mathscr{G}\left(\mu_{i}, \mu_{j}, \mu_{j}\right)\right\}\right),
$$

for all $k, n \in \mathbb{N}, 2 \leq k<n$. Knowing that

$$
\begin{aligned}
& \sup _{1 \leq i, j \leq n}\left\{\mathscr{G}\left(\mu_{i}, \mu_{j}, \mu_{j}\right)\right\} \\
& \leq \max \left\{\mathscr{G}\left(\mu_{1}, \mu_{2}, \mu_{2}\right), \mathscr{G}\left(\mu_{2}, \mu_{1}, \mu_{1}\right)\right\} \\
&+\sup _{2 \leq i, j \leq n}\left\{\mathscr{G}\left(\mu_{i}, \mu_{j}, \mu_{j}\right)\right\}
\end{aligned}
$$


and using (42) with $k=2$, we obtain

$$
\begin{aligned}
\sup _{1 \leq i, j \leq n}\left\{\mathscr{G}\left(\mu_{i}, \mu_{j}, \mu_{j}\right)\right\} \\
\leq \max \left\{\mathscr{G}\left(\mu_{1}, \mu_{2}, \mu_{2}\right), \mathscr{G}\left(\mu_{2}, \mu_{1}, \mu_{1}\right)\right\} \\
+\Phi\left(\sup _{1 \leq i, j \leq n}\left\{\mathscr{G}\left(\mu_{i}, \mu_{j}, \mu_{j}\right)\right\}\right) .
\end{aligned}
$$

Putting $x=\max \left\{\mathscr{G}\left(\mu_{1}, \mu_{2}, \mu_{2}\right), \mathscr{G}\left(\mu_{2}, \mu_{1}, \mu_{1}\right)\right\}$ and applying the property $\left(\phi_{5}\right)$ in $(44)$, there exists a $y(x)>0$ such that

$$
\sup _{1 \leq i, j \leq n}\left\{\mathscr{G}\left(\mu_{i}, \mu_{j}, \mu_{j}\right)\right\} \leq y(x) .
$$

Taking $k=2$ in (42) again, using the last relation, we get

$$
\sup _{2 \leq i, j \leq n}\left\{\mathscr{G}\left(\mu_{i}, \mu_{j}, \mu_{j}\right)\right\} \leq \Phi(y(x)) \text {. }
$$

Continuing this process, we obtain

$$
\sup _{k \leq i, j \leq n}\left\{\mathscr{G}\left(\mu_{i}, \mu_{j}, \mu_{j}\right)\right\} \leq \Phi^{k-1}(y(x))
$$

where $k, n \in \mathbb{N}, 2 \leq k<n$. Now, letting $k \rightarrow \infty$ and using the properties of $\Phi$, finally we show that $\left\{\mu_{i}\right\}$ is a Cauchy sequence; that is

$$
\lim _{k \rightarrow \infty} \sup _{k \leq i, j \leq n}\left\{\mathscr{G}\left(\mu_{i}, \mu_{j}, \mu_{j}\right)\right\} \leq \lim _{k \rightarrow \infty} \Phi^{k-1}(y(x))=0
$$

Using Lemma 14(ii), property $\left(\phi_{3}\right)$ can be replaced by $\left(\phi_{2}\right)$ or $\left(\phi_{4}\right)$.

Lemma 20. If $\Phi$ in (37) satisfies $\left(\phi_{6}\right)$ together with $\left(\phi_{2}\right)$ or $\left(\phi_{3}\right)$ or $\left(\phi_{4}\right)$, the sequence $\left\{\mu_{i}\right\}$ defined in (39) is a Cauchy sequence.

Proof. Using properties of $\Phi$ satisfying $\left(\phi_{6}\right)$ together with $\left(\phi_{4}\right)$, we prove by contradiction that

$$
\lim _{n \rightarrow \infty} \sup _{1 \leq i, j \leq n}\left\{\mathscr{G}\left(\mu_{i}, \mu_{j}, \mu_{j}\right)\right\}=\sup _{1 \leq i, j}\left\{\mathscr{G}\left(\mu_{i}, \mu_{j}, \mu_{j}\right)\right\}<\infty
$$

The assumption that (49) is not the case leads to

$$
\lim _{n \rightarrow \infty} \sup _{1 \leq i, j \leq n}\left\{\mathscr{G}\left(\mu_{i}, \mu_{j}, \mu_{j}\right)\right\}=\infty
$$

On the other hand, by $\left(\phi_{6}\right)$ and (44) we obtain

$$
\begin{aligned}
\infty= & \lim _{n \rightarrow \infty}\left(\sup _{1 \leq i, j \leq n}\left\{\mathscr{G}\left(\mu_{i}, \mu_{j}, \mu_{j}\right)\right\}\right. \\
& \left.-\phi\left(\sup _{1 \leq i, j \leq n}\left\{\mathscr{G}\left(\mu_{i}, \mu_{j}, \mu_{j}\right)\right\}\right)\right) \\
\leq & \max \left\{\mathscr{G}\left(\mu_{1}, \mu_{2}, \mu_{2}\right), \mathscr{G}\left(\mu_{2}, \mu_{1}, \mu_{1}\right)\right\},
\end{aligned}
$$

which is a contradiction implying that (49) is true. Now we define a decreasing sequence $\left\{x_{k}\right\} \subset(0, \infty)$ by

$$
x_{k}=\sup _{k \leq i, j}\left\{\mathscr{G}\left(\mu_{i}, \mu_{j}, \mu_{j}\right)\right\}
$$

and, using (41),

$$
x_{k} \leq \phi\left(x_{k-1}\right) \leq \cdots \leq \phi^{k-1}\left(x_{1}\right)
$$

Letting $k \rightarrow \infty$, finally we prove that

$$
\lim _{k \rightarrow \infty} \sup _{k \leq i, j}\left\{\mathscr{G}\left(\mu_{i}, \mu_{j}, \mu_{j}\right)\right\}=0
$$

So, $\left\{\mu_{i}\right\}$ is a Cauchy sequence. Using Lemma 14(ii), property $\left(\phi_{4}\right)$ can be replaced by $\left(\phi_{2}\right)$ or $\left(\phi_{3}\right)$.

Theorem 21. If all assumptions from Lemma 19 or from Lemma 20 are satisfied, then there exists a $\nu^{*} \in \mathscr{F}(X)$ such that $\nu^{*} \subseteq f_{i}\left(\nu^{*}\right)$ for all $i \in \mathbb{N}$.

Proof. In Lemma 19 or Lemma 20 it was proved that $\left\{\mu_{i}\right\}$ is a Cauchy sequence and, by the completeness of $(\mathscr{F}(X), \mathscr{G})$, $\lim _{i \rightarrow \infty} \mu_{i}=\mu^{*} \in \mathscr{F}(X)$. To prove that $\mu^{*} \subseteq f_{j}\left(\mu^{*}\right)$, we proceed as follows:

$$
\begin{gathered}
\mathscr{L}\left(\mu^{*}, f_{j}\left(\mu^{*}\right), f_{j}\left(\mu^{*}\right)\right) \\
\leq \lim _{i \rightarrow \infty} \mathscr{L}\left(\mu^{*}, \mu_{i}, \mu_{i}\right)+\lim _{i \rightarrow \infty} \mathscr{L}\left(\mu_{i}, f_{j}\left(\mu^{*}\right), f_{j}\left(\mu^{*}\right)\right) \\
=\lim _{i \rightarrow \infty} \mathscr{G}\left(f_{i}\left(\mu_{i-1}\right), f_{j}\left(\mu^{*}\right), f_{j}\left(\mu^{*}\right)\right) \\
\leq \lim _{i \rightarrow \infty} \Phi\left(\operatorname { m a x } \left\{\mathscr{G}\left(\mu_{i-1}, \mu^{*}, \mu^{*}\right),\right.\right. \\
\mathscr{L}\left(\mu_{i-1}, f_{i}\left(\mu_{i-1}\right), f_{i}\left(\mu_{i-1}\right)\right), \\
\mathscr{L}\left(\mu^{*}, f_{j}\left(\mu^{*}\right), f_{j}\left(\mu^{*}\right)\right), \\
\mathscr{L}\left(\mu_{i-1}, f_{j}\left(\mu^{*}\right), f_{j}\left(\mu^{*}\right)\right), \\
\left.\left.\mathscr{L}\left(\mu^{*}, f_{i}\left(\mu_{i-1}\right), f_{i}\left(\mu_{i-1}\right)\right)\right\}\right) \\
\leq \lim _{i \rightarrow \infty}\left(\operatorname { m a x } \left\{\mathscr{G}\left(\mu_{i-1}, \mu^{*}, \mu^{*}\right), \mathscr{L}\left(\mu_{i-1}, \mu_{i}, \mu_{i}\right),\right.\right. \\
\mathscr{L}\left(\mu^{*}, f_{j}\left(\mu^{*}\right), f_{j}\left(\mu^{*}\right)\right), \\
\mathscr{L}\left(\mu_{i-1}, f_{j}\left(\mu^{*}\right), f_{j}\left(\mu^{*}\right)\right), \\
\left.\left.\mathscr{L}\left(\mu^{*}, \mu_{i}, \mu_{i}\right)\right\}\right)
\end{gathered}
$$




$$
\begin{gathered}
\leq \Phi\left(\operatorname { m a x } \left\{\mathscr{L}\left(\mu^{*}, \mu^{*}, \mu^{*}\right), \mathscr{L}\left(\mu^{*}, \mu^{*}, \mu^{*}\right),\right.\right. \\
\mathscr{L}\left(\mu^{*}, f_{j}\left(\mu^{*}\right), f_{j}\left(\mu^{*}\right)\right), \\
\mathscr{L}\left(\mu^{*}, f_{j}\left(\mu^{*}\right), f_{j}\left(\mu^{*}\right)\right), \\
\left.\left.\mathscr{L}\left(\mu^{*}, \mu^{*}, \mu^{*}\right)\right\}\right) \\
=\Phi\left(\operatorname { m a x } \left\{0,0, \mathscr{L}\left(\mu^{*}, f_{j}\left(\mu^{*}\right), f_{j}\left(\mu^{*}\right)\right),\right.\right. \\
\left.\left.0+\mathscr{L}\left(\mu^{*}, f_{j}\left(\mu^{*}\right), f_{j}\left(\mu^{*}\right)\right), 0\right\}\right) \\
\leq \mathscr{L}\left(\mu^{*}, f_{j}\left(\mu^{*}\right), f_{j}\left(\mu^{*}\right)\right) .
\end{gathered}
$$

Hence, $\mathscr{L}\left(\mu^{*}, f_{j}\left(\mu^{*}\right), f_{j}\left(\mu^{*}\right)\right)=0 \Rightarrow \mu^{*} \subseteq f_{j}\left(\mu^{*}\right)$, for all $j \in \mathbb{N}$, what we had to prove.

Let $\varphi:[0, \infty)^{5} \rightarrow[0, \infty)$ be nondecreasing continuous from the right function with respect to each of the five variables such that

$$
\sum_{k \in \mathbb{N}} \varphi^{k}(t, t, t, 2 t, 0)<\infty,
$$

for all $t>0$. Obviously, for all $t>0$,

$$
\begin{gathered}
\lim _{k} \varphi^{k}(t, t, t, 2 t, 0)=0, \quad \varphi(t, t, t, 2 t, 0)<t, \\
\varphi(0,0,0,0,0)=0 .
\end{gathered}
$$

Further, let $(X, d)$ be a complete metric space, let $(\mathscr{F}(X), \mathscr{G})$ be related $G$-metric fuzzy space, and the family of selfmapings $\left\{f_{i}\right\}$ satisfy the next inequality:

$$
\begin{aligned}
\mathscr{G}\left(f_{i}(\nu), f_{j}(\mu), f_{j}(\mu)\right) \\
\leq \varphi\left(\mathscr{G}(\nu, \mu, \mu), \mathscr{L}\left(\nu, f_{i}(\nu), f_{i}(\nu)\right),\right. \\
\mathscr{L}\left(\mu, f_{j}(\mu), f_{j}(\mu)\right), \\
\\
\left.\mathscr{L}\left(\nu, f_{j}(\mu), f_{j}(\mu)\right), \mathscr{L}\left(\mu, f_{i}(\nu), f_{i}(\nu)\right)\right),
\end{aligned}
$$

for all $\mu, \nu \in \mathscr{F}(X)$ and all $i, j \in \mathbb{N}$.

Using the same arguments as in (39), the sequence $\left\{\mu_{i}\right\}$ is formed:

$$
\mu_{i+1} \subseteq f_{i+1}\left(\mu_{i}\right), \quad \forall i \in \mathbb{N},
$$

with property

$$
\mathscr{G}\left(\mu_{i}, \mu_{i+1}, \mu_{i+1}\right) \leq \mathscr{G}\left(f_{i}\left(\mu_{i-1}\right), f_{i+1}\left(\mu_{i}\right), f_{i+1}\left(\mu_{i}\right)\right) .
$$

Lemma 22. The sequence $\left\{\mu_{i}\right\}$ defined in (59) is a Cauchy sequence.

Proof. In order to prove that $\left\{\mu_{i}\right\}$ is a Cauchy sequence, we consider the sequence $\left\{\mathscr{G}\left(\mu_{i}, \mu_{i+1}, \mu_{i+1}\right)\right\}$. Using relations
(58) and (60) and the implication $\mu_{i} \subseteq f_{i}\left(\mu_{i-1}\right) \Rightarrow \mathscr{L}\left(\mu_{i}\right.$, $\left.f_{i}\left(\mu_{i-1}\right), f_{i}\left(\mu_{i-1}\right)\right)=0$, we get

$$
\begin{aligned}
& \mathscr{G}\left(\mu_{i}, \mu_{i+1}, \mu_{i+1}\right) \\
& \leq \mathscr{G}\left(f_{i}\left(\mu_{i-1}\right), f_{i+1}\left(\mu_{i}\right), f_{i+1}\left(\mu_{i}\right)\right) \\
& \leq \varphi\left(\mathscr{G}\left(\mu_{i-1}, \mu_{i}, \mu_{i}\right), \mathscr{L}\left(\mu_{i-1}, f_{i}\left(\mu_{i-1}\right), f_{i}\left(\mu_{i-1}\right)\right),\right. \\
& \mathscr{L}\left(\mu_{i}, f_{i+1}\left(\mu_{i}\right), f_{i+1}\left(\mu_{i}\right)\right), \\
& \mathscr{L}\left(\mu_{i-1}, f_{i+1}\left(\mu_{i}\right), f_{i+1}\left(\mu_{i}\right)\right) \text {, } \\
& \left.\mathscr{L}\left(\mu_{i}, f_{i}\left(\mu_{i-1}\right), f_{i}\left(\mu_{i-1}\right)\right)\right) \\
& \leq \varphi\left(\mathscr{G}\left(\mu_{i-1}, \mu_{i}, \mu_{i}\right), \mathscr{L}\left(\mu_{i-1}, \mu_{i}, \mu_{i}\right),\right. \\
& \left.\mathscr{L}\left(\mu_{i}, \mu_{i+1}, \mu_{i+1}\right), \mathscr{L}\left(\mu_{i-1}, \mu_{i+1}, \mu_{i+1}\right), 0\right) \\
& \leq \varphi\left(\mathscr{G}\left(\mu_{i-1}, \mu_{i}, \mu_{i}\right), \mathscr{G}\left(\mu_{i-1}, \mu_{i}, \mu_{i}\right), \mathscr{G}\left(\mu_{i}, \mu_{i+1}, \mu_{i+1}\right)\right. \text {, } \\
& \left.\mathscr{G}\left(\mu_{i-1}, \mu_{i+1}, \mu_{i+1}\right), 0\right) \text {. }
\end{aligned}
$$

Since $\mathscr{G}\left(\mu_{i-1}, \mu_{i+1}, \mu_{i+1}\right) \leq \mathscr{G}\left(\mu_{i-1}, \mu_{i}, \mu_{i}\right)+\mathscr{G}\left(\mu_{i}, \mu_{i+1}, \mu_{i+1}\right)$, the next relation holds:

$$
\begin{aligned}
& \mathscr{G}\left(\mu_{i}, \mu_{i+1}, \mu_{i+1}\right) \\
& \leq \varphi( \mathscr{G}\left(\mu_{i-1}, \mu_{i}, \mu_{i}\right), \\
& \mathscr{G}\left(\mu_{i-1}, \mu_{i}, \mu_{i}\right), \mathscr{G}\left(\mu_{i}, \mu_{i+1}, \mu_{i+1}\right), \\
&\left.\mathscr{G}\left(\mu_{i-1}, \mu_{i}, \mu_{i}\right)+\mathscr{G}\left(\mu_{i}, \mu_{i+1}, \mu_{i+1}\right), 0\right) .
\end{aligned}
$$

The assumption $\mathscr{G}\left(\mu_{i}, \mu_{i+1}, \mu_{i+1}\right) \quad \npreceq \mathscr{G}\left(\mu_{i-1}, \mu_{i}, \mu_{i}\right)$, which is equivalent to $\mathscr{G}\left(\mu_{i}, \mu_{i+1}, \mu_{i+1}\right)>\mathscr{G}\left(\mu_{i-1}, \mu_{i}, \mu_{i}\right)$, leads to inequality

$$
\begin{aligned}
\mathscr{G}\left(\mu_{i}, \mu_{i+1}, \mu_{i+1}\right) \\
\leq \varphi\left(\mathscr{G}\left(\mu_{i}, \mu_{i+1}, \mu_{i+1}\right), \mathscr{G}\left(\mu_{i}, \mu_{i+1}, \mu_{i+1}\right),\right. \\
\left.\quad \mathscr{G}\left(\mu_{i}, \mu_{i+1}, \mu_{i+1}\right), 2 \mathscr{G}\left(\mu_{i}, \mu_{i+1}, \mu_{i+1}\right), 0\right) \\
<\mathscr{G}\left(\mu_{i}, \mu_{i+1}, \mu_{i+1}\right),
\end{aligned}
$$

which is a contradiction. In the last transformation we used nondecreasingness of $\phi$ and the property $\varphi(t, t, t, 2 t, 0)<$ $t, t \in \mathbb{R}^{+}$. Hence, $\mathscr{G}\left(\mu_{i}, \mu_{i+1}, \mu_{i+1}\right) \leq \mathscr{G}\left(\mu_{i-1}, \mu_{i}, \mu_{i}\right)$. With notation $\mathscr{G}\left(\mu_{i-1}, \mu_{i}, \mu_{i}\right)=t_{i}$, we have

$$
t_{i+1} \leq \varphi\left(t_{i}, t_{i}, t_{i}, 2 t_{i}, 0\right)<t_{i} \leq \cdots \leq \varphi^{i}\left(t_{1}, t_{1}, t_{1}, 2 t_{1}, 0\right) .
$$

If $\mu_{0}=\mu_{1}$, then $t_{1}=\mathscr{G}\left(\mu_{0}, \mu_{1}, \mu_{1}\right)=0$. Since $\varphi(0,0,0,0,0)=0$, from inequality $(64)$ we get $\left(\mu_{0}=\mu_{1}\right) \Rightarrow$ $\left(\mu_{1}=\mu_{2}\right) \Rightarrow \cdots \Rightarrow\left(\mu_{i-1}=\mu_{i}\right) \Rightarrow \cdots$. Further, $\mu_{0}=\mu_{i} \subseteq$ $f_{i}\left(\mu_{i-1}\right)=f_{i}\left(\mu_{0}\right)$, which means that $\mu^{*}=\mu_{0}$ and the proof is completed. 
If $\mu_{0} \neq \mu_{1}$, we prove that $\lim _{i \rightarrow \infty} \mathscr{G}\left(\mu_{i}, \mu_{j}, \mu_{j}\right)=0$ whenever $i<j$ :

$$
\begin{aligned}
& \mathscr{G}\left(\mu_{i}, \mu_{j}, \mu_{j}\right) \\
& \leq \mathscr{G}\left(\mu_{i}, \mu_{i+1}, \mu_{i+1}\right)+\mathscr{G}\left(\mu_{i+1}, \mu_{i+2}, \mu_{i+2}\right) \\
&+\cdots+\mathscr{G}\left(\mu_{j-1}, \mu_{j}, \mu_{j}\right) \\
&= \sum_{k=i}^{j-1} \mathscr{G}\left(\mu_{k}, \mu_{k+1}, \mu_{k+1}\right) \\
&= \sum_{k=i}^{j-1} t_{k+1} \leq \sum_{k=i}^{j-1} \varphi^{k}\left(t_{1}, t_{1}, t_{1}, 2 t_{1}, 0\right),
\end{aligned}
$$

and, since $t_{1}=\mathscr{G}\left(\mu_{0}, \mu_{1}, \mu_{1}\right)>0, \lim _{i \rightarrow \infty} \mathscr{G}\left(\mu_{i}, \mu_{j}, \mu_{j}\right)=$ 0 . Also, $\mathscr{G}\left(\mu_{j}, \mu_{i}, \mu_{i}\right) \leq 2 \mathscr{G}\left(\mu_{i}, \mu_{j}, \mu_{j}\right) \rightarrow 0$. So, we have proved that $\left\{\mu_{i}\right\}$ is a Cauchy sequence and, consequently, there exists a $\mu^{*} \in \mathscr{F}(X)$ such that $\lim _{i \rightarrow \infty} \mu_{i}=\mu^{*}$; that is, $\lim _{i \rightarrow \infty} \mathscr{G}\left(\mu_{i}, \mu^{*}, \mu^{*}\right)=\lim _{i \rightarrow \infty} \mathscr{G}\left(\mu^{*}, \mu_{i}, \mu_{i}\right)=0$.

Theorem 23. If all assumptions from the previous lemma are satisfied, then there exists a $v^{*} \in \mathscr{F}(X)$ such that $v^{*} \subseteq f_{i}\left(v^{*}\right)$ for all $i \in \mathbb{N}$.

Proof. If $\mathscr{L}\left(\mu^{*}, f_{j}\left(\mu^{*}\right), f_{j}\left(\mu^{*}\right)\right)=0$ for all $j \in \mathbb{N}$, then $\mu^{*} \subseteq f_{j}\left(\mu^{*}\right)$. So, assume that, for some $j \in \mathbb{N}, \mathscr{L}\left(\mu^{*}\right.$, $\left.f_{j}\left(\mu^{*}\right), f_{j}\left(\mu^{*}\right)\right)>0$. Then

$$
\begin{gathered}
\mathscr{L}\left(\mu^{*}, f_{j}\left(\mu^{*}\right), f_{j}\left(\mu^{*}\right)\right) \\
\leq \lim _{i \rightarrow \infty} \mathscr{L}\left(\mu^{*}, \mu_{i}, \mu_{i}\right)+\lim _{i \rightarrow \infty} \mathscr{L}\left(\mu_{i}, f_{j}\left(\mu^{*}\right), f_{j}\left(\mu^{*}\right)\right) \\
\leq \lim _{i \rightarrow \infty} \mathscr{L}\left(f_{i}\left(\mu_{i-1}\right), f_{j}\left(\mu^{*}\right), f_{j}\left(\mu^{*}\right)\right) \\
\leq \lim _{i \rightarrow \infty} \mathscr{L}\left(f_{i}\left(\mu_{i-1}\right), f_{j}\left(\mu^{*}\right), f_{j}\left(\mu^{*}\right)\right) \\
\leq \lim _{i \rightarrow \infty} \varphi\left(\mathscr{G}\left(\mu_{i-1}, \mu^{*}, \mu^{*}\right), \mathscr{L}\left(\mu_{i-1}, f_{i}\left(\mu_{i-1}\right), f_{i}\left(\mu_{i-1}\right)\right),\right. \\
\mathscr{L}\left(\mu^{*}, f_{j}\left(\mu^{*}\right), f_{j}\left(\mu^{*}\right)\right), \\
\mathscr{L}\left(\mu_{i-1}, f_{j}\left(\mu^{*}\right), f_{j}\left(\mu^{*}\right)\right), \\
\left.\mathscr{L}\left(\mu^{*}, f_{i}\left(\mu_{i-1}\right), f_{i}\left(\mu_{i-1}\right)\right)\right) \\
\leq \lim _{i \rightarrow \infty} \varphi\left(\mathscr{G}\left(\mu_{i-1}, \mu^{*}, \mu^{*}\right), \mathscr{L}\left(\mu_{i-1}, \mu_{i}, \mu_{i}\right),\right. \\
\mathscr{L}\left(\mu^{*}, f_{j}\left(\mu^{*}\right), f_{j}\left(\mu^{*}\right)\right), \\
\mathscr{L}\left(\mu_{i-1}, f_{j}\left(\mu^{*}\right), f_{j}\left(\mu^{*}\right)\right), \\
\left.\mathscr{L}\left(\mu^{*}, \mu_{i}, \mu_{i}\right)\right) .
\end{gathered}
$$

If we put $a=\mathscr{L}\left(\mu^{*}, f_{j}\left(\mu^{*}\right), f_{j}\left(\mu^{*}\right)\right)>0$, since $\lim _{i \rightarrow \infty} \mu_{i}=$ $\mu^{*}$, for every $\varepsilon \in(0, a)$ there exists an $i_{0}=\max \left\{i_{1}, i_{2}, i_{3}, i_{4}\right\}$, where (i) $\mathscr{G}\left(\mu_{i-1}, \mu^{*}, \mu^{*}\right)<\varepsilon<a$ for all $i>i_{1}$,

(ii) $\mathscr{L}\left(\mu_{i-1}, \mu_{i}, \mu_{i}\right)<\varepsilon<a$ for all $i>i_{2}$,

(iii) $\mathscr{L}\left(\mu_{i-1}, f_{j}\left(\mu^{*}\right), f_{j}\left(\mu^{*}\right)\right) \leq \mathscr{L}\left(\mu_{i-1}, \mu_{i}, \mu_{i}\right)+\mathscr{L}\left(\mu_{i}\right.$, $\left.f_{j}\left(\mu^{*}\right), f_{j}\left(\mu^{*}\right)\right)<(\varepsilon / 2)+a+(\varepsilon / 2)<2 a$, for all $i>i_{3}$,

(iv) $\mathscr{L}\left(\mu^{*}, \mu_{i}, \mu_{i}\right)<\varepsilon$ for all $i>i_{4}$.

Now, relation (66) becomes

$$
\begin{aligned}
\mathscr{L}\left(\mu^{*}, f_{j}\left(\mu^{*}\right), f_{j}\left(\mu^{*}\right)\right) & \leq \varphi(\varepsilon, \varepsilon, a, \varepsilon+a, \varepsilon) \\
& \leq \varphi(a, a, a, 2 a, \varepsilon)
\end{aligned}
$$

and, letting $\varepsilon \rightarrow 0$, we get $a \leq \varphi(a, a, a, 2 a, 0)<a$, where $a=\mathscr{L}\left(\mu^{*}, f_{j}\left(\mu^{*}\right), f_{j}\left(\mu^{*}\right)\right)$. Hence, $\mathscr{L}\left(\mu^{*}, f_{j}\left(\mu^{*}\right), f_{j}\left(\mu^{*}\right)\right)=$ $0 \Rightarrow \mu^{*} \subseteq f_{j}\left(\mu^{*}\right)$, for all $j \in \mathbb{N}$, what we had to prove.

\section{Conflict of Interests}

The authors declare that there is no conflict of interests regarding the publication of this paper.

\section{Acknowledgment}

This work was partially supported by Ministarstvo Nauke i Životne Sredine Republike Srbije.

\section{References}

[1] O. Kaleva and S. Seikkala, "On fuzzy metric spaces," Fuzzy Sets and Systems, vol. 12, no. 3, pp. 215-229, 1984.

[2] W.-S. Du, "The existence of cone critical point and common fixed point with applications," Journal of Applied Mathematics, vol. 2011, Article ID 985797, 22 pages, 2011.

[3] S. N. Ješić, N. A. Babačev, and R. M. Nikolić, "A common fixed point theorem in fuzzy metric spaces with nonlinear contractive type condition defined using $\Phi$-function," Abstract and Applied Analysis, vol. 2013, Article ID 273872, 6 pages, 2013.

[4] S. Manro, S. Kumar, S. S. Bhatia, and K. Tas, "Common fixed point theorems in modified intuitionistic fuzzy metric spaces," Journal of Applied Mathematics, vol. 2013, Article ID 189321, 13 pages, 2013.

[5] K. P. R. Rao and K. R. K. Rao, "A triple fixed point theorem for multimap in a hausdorff fuzzy metric space," Journal of Mathematics, vol. 2013, Article ID 812153, 6 pages, 2013.

[6] Y. Shen, D. Qiu, and W. Chen, "On convergence of fixed points in fuzzy metric spaces," Abstract and Applied Analysis, vol. 2013, Article ID 135202, 6 pages, 2013.

[7] J. Zhu, Y. Wang, and S. M. Kang, "Common fixed point theorems of new contractive conditions in fuzzy metric spaces," Journal of Applied Mathematics, vol. 2013, Article ID 145190, 9 pages, 2013.

[8] Z. Mustafa and B. Sims, "A new approach to generalized metric spaces," Journal of Nonlinear and Convex Analysis, vol. 7, no. 2, pp. 289-297, 2006.

[9] A. Kaewcharoen and A. Kaewkhao, "Common fixed points for single-valued and multi-valued mappings in G-metric spaces," International Journal of Mathematical Analysis, vol. 5, no. 33-36, pp. 1775-1790, 2011. 
[10] L. Zhu, C. X. Zhu, and C. F. Chen, "Common fixed point theorems for fuzzy mappings in G-metric spaces," Fixed Point Theory and Aplications, vol. 2012, article 159, 2012.

[11] T. Kamran, "Common fixed points theorems for fuzzy mappings," Chaos, Solitons and Fractals, vol. 38, no. 5, pp. 1378-1382, 2008.

[12] D. Qiu, L. Shu, and J. Guan, "Common fixed point theorems for fuzzy mappings under $\Phi$-contraction condition," Chaos, Solitons and Fractals, vol. 41, no. 1, pp. 360-367, 2009.

[13] M. Jleli and B. Samet, "Remarks on G-metric spaces and fixed point theorems," Fixed Point Theory and Applications, vol. 2012, article 210, 2012.

[14] B. Samet, C. Vetro, and F. Vetro, "Remarks on G-metric spaces," International Journal of Analysis, vol. 2013, Article ID 917158, 6 pages, 2013.

[15] Z. Mustafa, H. Obiedat, and F. Awawdeh, "Some fixed point theorem for mapping on complete G-metric spaces," Fixed Point Theory and Applications, vol. 2008, Article ID 189870, 12 pages, 2008.

[16] E. Karapinar and R. Agarval, "Further remarks on G-metric spaces," Fixed Point Theory and Applications, vol. 2013, article 154, 2013.

[17] L. Gajić and Z. Lozanov-Crvenković, "On mappings with contractive iterate at a point in generalized metric spaces," Fixed Point Theory and Applications, vol. 2010, Article ID 458086, 16 pages, 2010.

[18] Lj. Gajić and M. Stojaković, "On Ćirić generalization of mappings with a contractive iterate at a point in $G$-metric spaces," Applied Mathematics and Computation, vol. 219, no. 1, pp. 435441, 2012.

[19] Z. Mustafa, W. Shatanawi, and M. Bataineh, "Existence of fixed point results in G-metric spaces," International Journal of Mathematics and Mathematical Sciences, vol. 2009, Article ID 283028, 10 pages, 2009.

[20] Z. Mustafa and B. Sims, "Fixed point theorems for contractive mappings in complete G-metric spaces," Fixed Point Theory and Applications, vol. 2009, Article ID 917175, 10 pages, 2009.

[21] Sh. Rezapour, R. H. Haghi, and N. Shahzad, "Some notes on fixed points of quasi-contraction maps," Applied Mathematics Letters, vol. 23, no. 4, pp. 498-502, 2010.

[22] W. Shatanawi, "Fixed point theory for contractive mappings satisfying $\Phi$-maps in G-metric spaces," Fixed Point Theory and Applications, vol. 2010, Article ID 181650, 9 pages, 2010. 


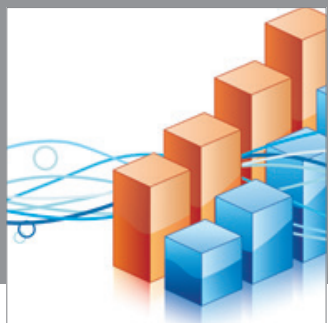

Advances in

Operations Research

mansans

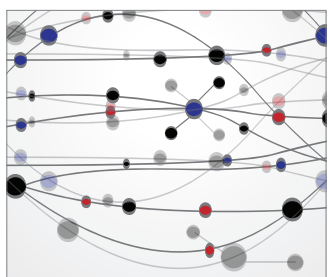

The Scientific World Journal
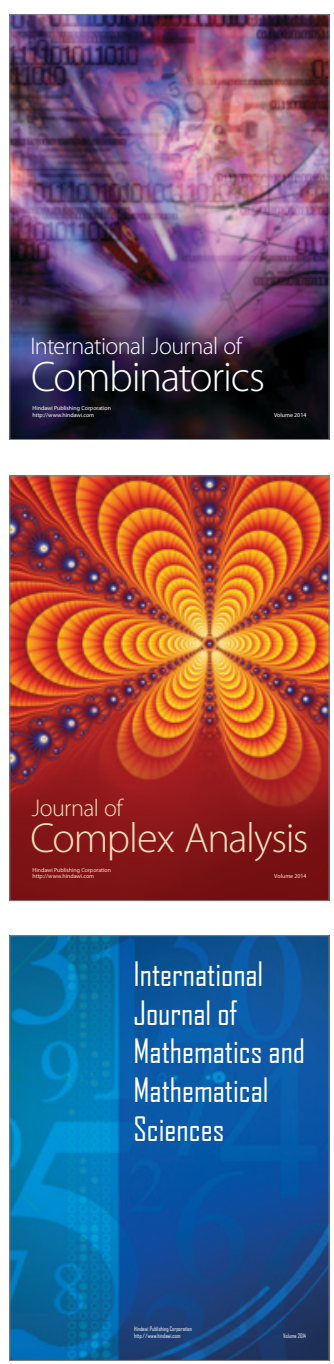
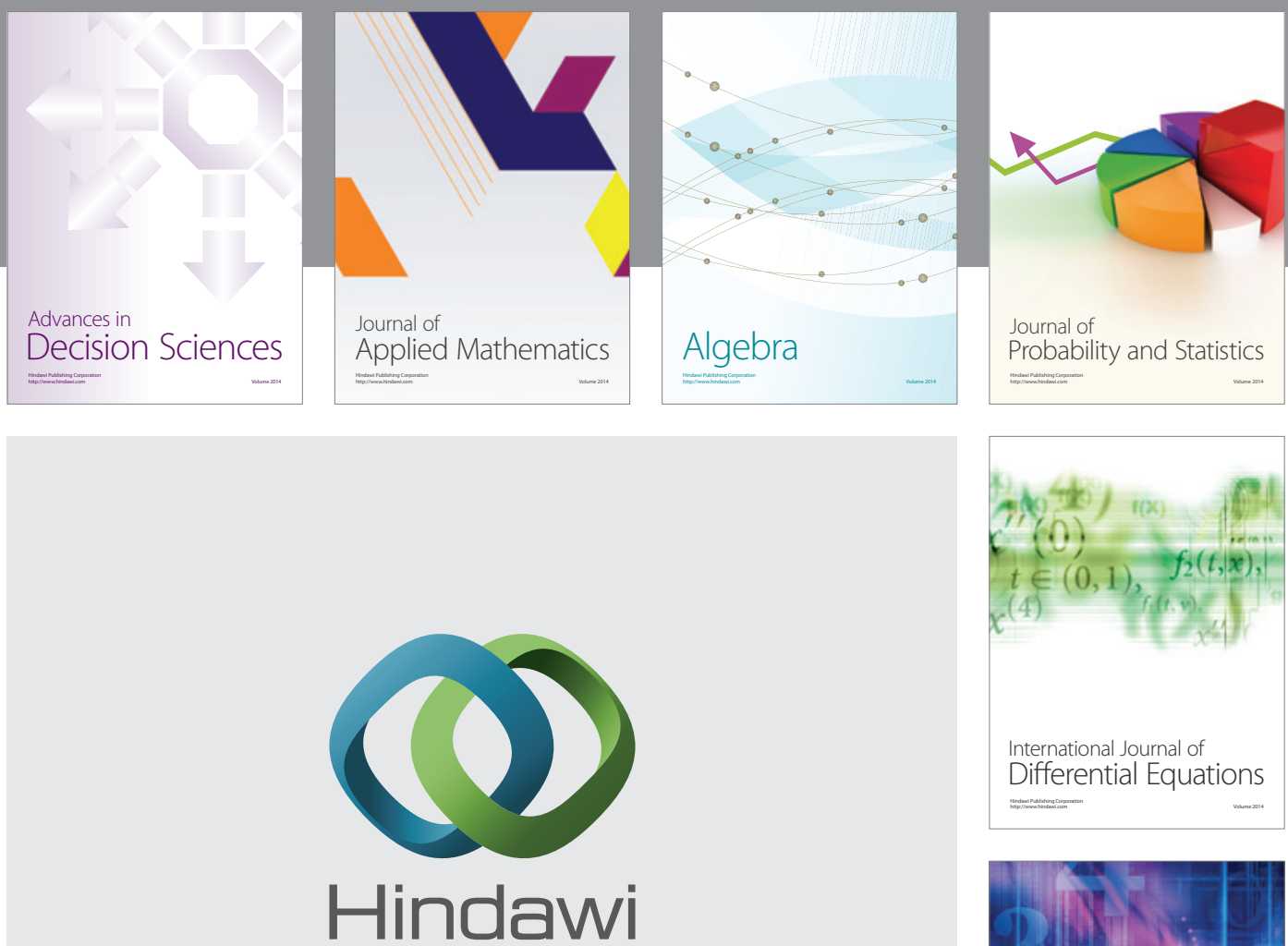

Submit your manuscripts at http://www.hindawi.com
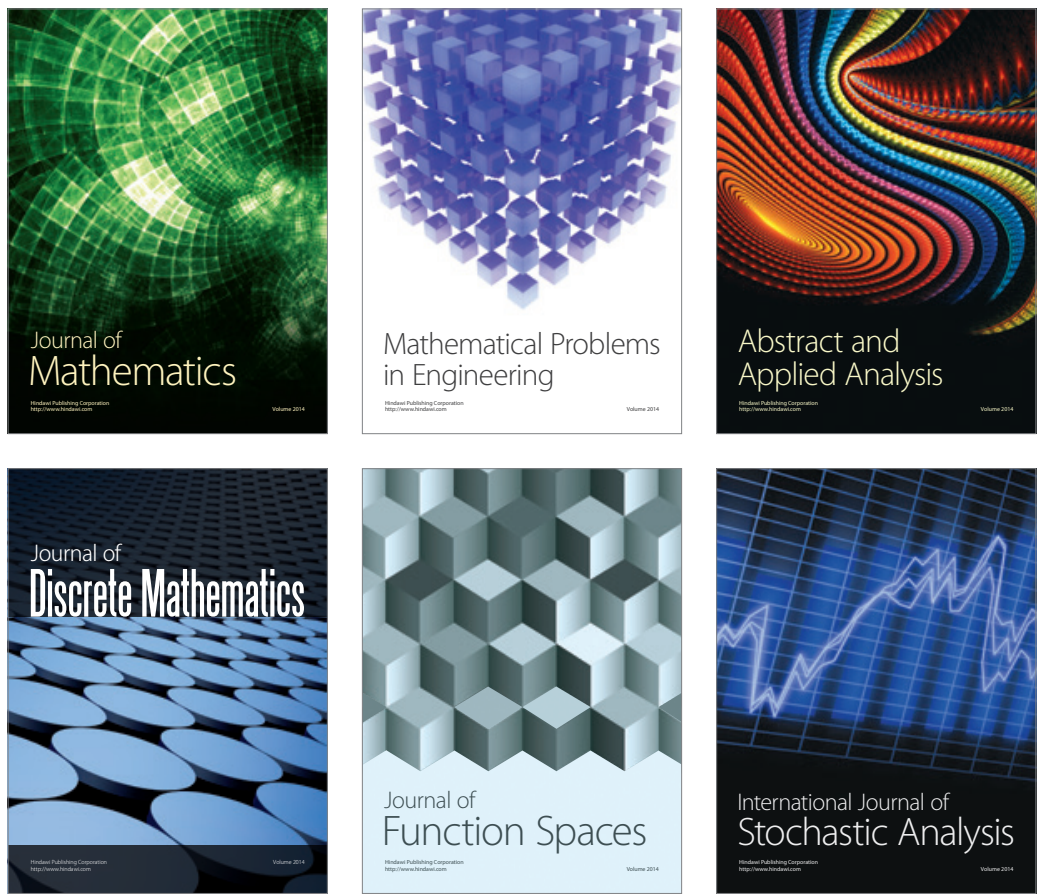

Journal of

Function Spaces

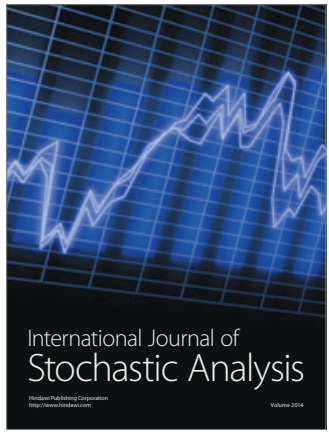

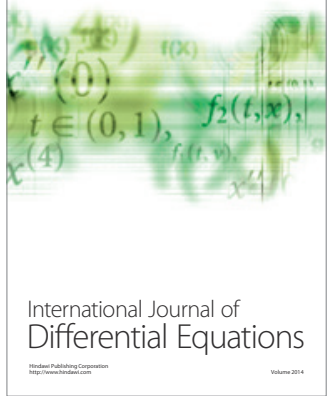
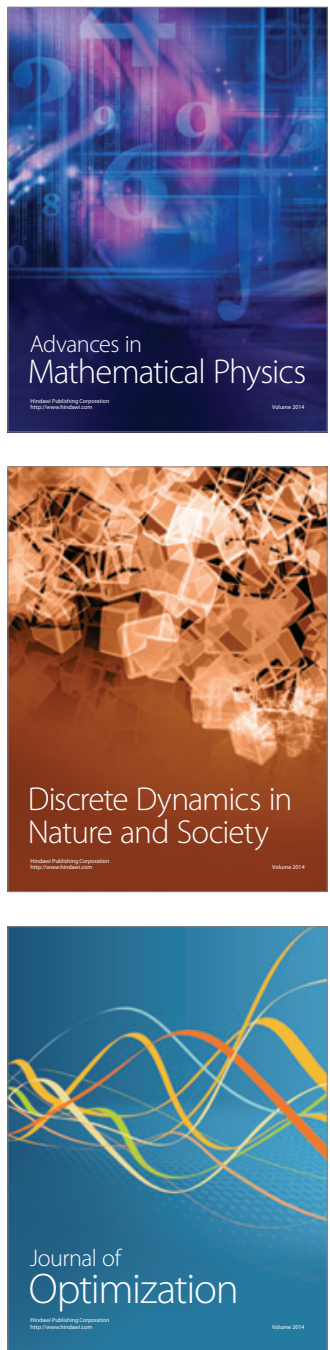